\title{
The Performance Investigation of Cooperative Multicast with CP Combining in Hexagonal Network and Small Cell Network
}

\author{
Jiachen Zhang ${ }^{\mathrm{a}}$, Yuanyuan $\mathrm{Li}^{\mathrm{b}}$, Xiaotong $\mathrm{Han}^{\mathrm{c}}$ \\ College of Software, Jilin University, Changchun, 130012, China \\ a zhangjc@jlu.edu.cn; b258030084@qq.com;c 164209273@qq.com;
}

Keywords: cooperative multicast, small cell network, hexagonal network, energy efficiency, random network

\begin{abstract}
With the rapid development of broadband mobile data services, small cell networks are more and more popular to enable high capacity wireless access. Unplanned small cells network, which is described as random network (RN), brings new characteristic different with conventional hexagonal network (HN). This paper mainly investigated the performance of cooperative multicast in $\mathrm{HN}$ and $\mathrm{RN}$ respectively, where $\mathrm{CP}$ combining signal processing is employed. The tractable expressions about the coverage and signal to noise ratio (SNR) distribution of cooperative multicast are derived. It is illustrated by analyses great gain is obtained through cooperation in both $\mathrm{HN}$ and $\mathrm{RN}$ and the BS power needed to ensure $95 \%$ coverage reduced by about $30 \%$ when only two BSs are employed to cooperative multicast. Since, given the same BS transmission power and BS distribution density, $\mathrm{HN}$ will be energy efficient than $\mathrm{RN}$. Therefore a larger number of BSs with low transmission power should be deployed in RN so that it is possible to be energy efficient as $\mathrm{HN}$.
\end{abstract}

\section{Introduction}

With the rapid development of mobile applications, high data rate is needed to meet the demands of subscribes. It is expected that the number of mobile broadband subscriptions will be 9 billion by the end of $2018^{[1]}$ and the data traffic will grow over $300 \%$ by 2017 to 21 Exabyte ${ }^{[2]}$, which presents great challenges to the cellular networks. New technologies are needed to enable high data rate access. Recently years, small cell network shows great talent to meet the capacity demands and had attracted lots of attention in both industrial and academy. Different with conventional hexagonal network $(\mathrm{HN})$, small cell network is based on random network $(\mathrm{HN})$ topologies and Poisson point process (PPP) is widely used to mode the distribution of small cells ${ }^{[3-5]}$. Paper ${ }^{[3]}$ firstly compared the performance of $\mathrm{HN}$ and $\mathrm{HN}$, the analyzes illustrated that $\mathrm{RN}$ is the same accurate as $\mathrm{HN}$ to describe the real network and it is believed that PPP model will be much more suitable to capture the characteristic of $4 \mathrm{G}$ network ${ }^{[4]}{ }^{[5]}$ studied the spectrum allocation and femto access polices in two-tier RN ${ }^{[5]}$ and the corresponding success probability for each tier is derived. The energy efficiency problem in $\mathrm{RN}$ is studied in ${ }^{[6]}$ where a tradeoff scheme of energy efficient cellular networks with small cells is proposed. These papers mainly pay much attention to single cell and didn't consider the cooperation of BS. Paper ${ }^{[7]}$ investigated the outage probability of multi-cell CoMP network with zero force beamforming technology. A user-centric adaptive clustering method is proposed in ${ }^{[8]}$, which is outperforms than static clustering scheme ${ }^{[9]}$. In summary, a few literatures investigated the cooperative in $\mathrm{RN}$ by so far.

Moreover, It is noted that mobile video has exceeded half of the total mobile data traffic in 2011 [10] and is expected to grow with a speed of $60 \%$. As one spectral efficient method for video services, multicast transmission plays a key role in cellular networks. It had been defined by the third generation partnership project (3GPP) ${ }^{[11]}$. The signal processing for BS cooperative multicast is different with unicast since many cooperative BSs multicast the same message simultaneously and the mobile station (MS) could receive multiple copies of the signal from different BSs. Assuming the $\mathrm{CP}$ is long enough to combine all the signals arriving within the cyclic prefix $(\mathrm{CP})$ duration, thus a stronger signal could be constructed in BS cooperative multicast, which is known as $\mathrm{CP}$ combining. 
Therefore, this paper focuses on multicast service in $\mathrm{RN}$ aiming to provide the same performance as that in $\mathrm{HN}$. We analyze the performance of BS cooperative multicast, where one MS is served by more than one BSs and the CP combining signal processing is employed. The close expression about the coverage and signal to noise ratio (SNR) distribution function in RN and HN are obtained respectively, which significantly simplified the complex simulations since the influence of different parameters could be easily presented by this expression.

The rest of the paper is organized as follows. Section 2 describes system model as well as CP Combining s ignal processing. Next, in Section 3, coverage performance analysis is carried out with BS cooperative multicast. Section 4 presents numerical results. Finally, conclusions are drawn in Section 5 .

\section{System description}

An orthogonal frequency division multiplexing (OFDM) based downlink transmission cellular system is investigated, where a single antenna is assumed ${ }^{[12]}$. Suppose there are $N$ cooperative multicast BSs and they all transmit the same multicast data simultaneously with the power $P_{B S}$, thus one MS could receive multiple copies of the signals. Moreover, assuming that the length of CP is longer than the maximum delay of the equivalent multipath channel, then all the signals arriving within the CP duration could be added up to construct a stronger signal. Therefore after synchronization and FFT at the destination, the received signal at MS $k$ is given by

$$
\begin{aligned}
Y_{k} & =\sum_{i=1}^{N} \sqrt{P_{B S}} \cdot A_{1} \cdot D_{i, k}^{-\gamma} H_{i, k} x+\eta_{k} \\
& =\sqrt{P_{B S}} H_{k} x+\eta_{k}
\end{aligned}
$$

where $A_{1} \cdot D_{i, k}^{-\gamma}$ represents the path loss from the BS $i$ to MS $k, A_{1}$ is a constant, ${ }^{D_{i, k}}$ stands for the distance between BS $i$ and MS $k$ and $\gamma$ is the path loss parameter. ${ }_{i, k}$ is the Rayleigh channel fading with an average power of 1 and follows systemic complex Gaussian distribution. $x$ represents the transmitted symbol with unit power and $\eta_{k}$ is the zero mean Gaussian noise with a variance of $\sigma^{2}=N_{0} B$, where $N_{0}$ is the power spectrum density of the Gaussian noise and $B$ is the cellular system bandwidth. Moreover, $H_{k}=\sum_{i=1}^{N} A_{1} \cdot D_{i, k}^{-\gamma} \cdot H_{i, k}$ is the channel impact from all the cooperative BSs including path loss and relay fading. Therefore, the SNR of $Y_{k}$ is given by $S N R_{k}=P_{B S}\left|H_{k}\right|^{2} / \sigma^{2}$.

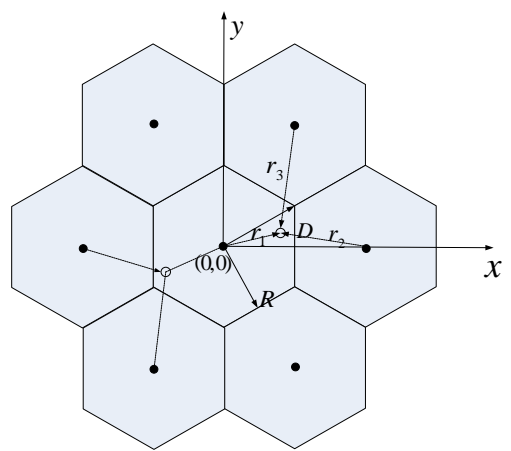

(a) Hexagonal Network

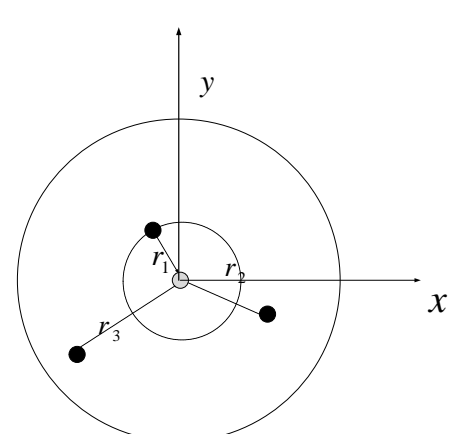

(b) Random Network

Figure 1. System Description of Hexagonal Network and Random Network

Assume that the multicast data rata is $R_{m}$, than the required $S N R_{m}$ can be obtained as $S N R_{m}=2^{R_{m} / B}-1$ according to Shannon theory. Therefore, the SNR of received signal $S N R_{k}$ must 
be no less than ${ }^{S N R_{m}}$ such that MS k could successfully decode the multicast data. Moreover, it is further supposed that all MSs are uniformly distributed in the hexagonal cell with a radius of $R$, and we model the small cell distribution with PPP of density, which represents the average number of small cell on unit area. Since the coverage area of one cell with radius $R$ is $6 R^{2} / \sqrt{3}$ in $\mathrm{HN}^{[13]}$, the parameter $\lambda$ is set to be $\left(\frac{6 R^{2}}{\sqrt{3}}\right)^{-1}$ in RN for purpose of comparison with HN.

\section{Bs cooperative multicast}

\section{Hexagonal network.}

The coverage of cooperative multicast is much more complicated due to the $\mathrm{CP}$ combining signal processing. It is assumed that one MS $k$ is served by the nearest N BS which are labeled by BS 1 to BS N, as shown in Fig 1(a), the success probability of MS $k$ is impact by the distance vector $z=\left\{r_{1}, r_{2}, \ldots r_{N}\right\}$ where ${ }^{r_{i}}$ stands for the distance between MS $\mathrm{k}$ and the $i$ th cooperative BS and meet $r_{1}<r_{2}<r_{3} \ldots<r_{N}$. Thus as described before, the success probability of MS $\mathrm{k}$ could be presented as:

$$
P\left(S N R_{k} \geq S N R_{m}\right)=\exp \left(-\frac{S N R_{m} \cdot \sigma^{2}}{P_{B S} \cdot \sum_{i=1}^{N} A_{1} \cdot r_{i}^{-\gamma}}\right)
$$

Next, consider the fact that the hexagonal cell is systematic, the average success probability (coverage) in the cell is the same with that of a triangle area, such as Region D (as shown in Fig1 (a)). Assuming that the position of BS 1 is set to be the origin $(0,0)$ and the axis are built as Fig 1 (a), the other cooperative BS coordinates could easily be obtained. So for one MS of coordinates (x, $\mathrm{y})$, the distance between MS and cooperative BS $i r_{i}$ is a function of $x$ and ${ }^{y}$ and thus the average success probability of MS which is uniformly distrusted in Region D could be given by:

$$
\begin{aligned}
& S_{H N}^{C}=\iint_{D} P\left(S N R_{k} \geq S N R_{m}\right) \cdot f_{D}(x, y) d x d y \\
& =\iint_{D} \exp \left(-\frac{S N R_{m} \cdot \sigma_{n o i}^{2}}{P_{B S} \cdot A_{1} \cdot \sum_{i=1}^{N} r_{i}(x, y)^{-\gamma}}\right) \cdot\left(\frac{R^{2}}{2 \sqrt{3}}\right)^{-1} d x d y
\end{aligned}
$$

\footnotetext{
where $f_{D}(x, y)=\left(\frac{R^{2}}{2 \sqrt{3}}\right)^{-1}$ is

is the pdf of one MS with coordinate $(x, y)$ in region D. Since MS is uniformly distributed in region $\mathrm{D}$, the pdf is a constant and is only related with the area of region $\mathrm{D}$. Thus the coverage of cooperative multicast in $\mathrm{HN}$ is derived.
}

\section{Random network.}

This subsection focuses on average success probability of MS in random network. With the same assumption that MS $k$ is served by the nearest $\mathrm{N}$ BSs and the distance vector is $z=\left\{r_{1}, r_{2}, r_{3}, \ldots, r_{N}\right\}$, where $r_{1}<r_{2}<r_{3}<\ldots<r_{N}$, therefore the average success probability in the cell could be expressed as:

$$
S_{R N}^{C}=\int P\left(S N R_{k} \geq S N R_{m}\right) f(z) d z
$$

where $f(z)$ is the joint probability distribution function of vector $\mathrm{z}$ and the key is to obtain the joint pdf of the distance vector $z$. 
According with the property of PPP ${ }^{[14]}$, the joint pdf of $\left\{r_{1}, r_{2}, \ldots, r_{N}\right\}$ could be given by:

$$
f\left(r_{1}, r_{2}, \ldots, r_{N}\right)=\exp \left(-\lambda \pi r_{N}^{2}\right)(2 \pi \lambda)^{N} \prod_{i=1}^{N} r_{i}
$$

Therefore, the average success probability could be given by:

$$
\begin{aligned}
& S_{R N}^{C}=\int P\left(S N R_{k} \geq S N R_{m}\right) f(z) d z \\
& =\int_{r_{N-1}}^{\infty} \int_{r_{N-2}}^{\infty} \ldots \int_{0}^{\infty} \exp \left(-\frac{S N R_{m} \cdot \sigma_{n o i}^{2}}{P_{B S} \cdot A_{1} \cdot \sum_{i=1}^{N} r_{i}^{-\gamma}}\right) \exp \left(-\lambda \pi r_{N}^{2}\right)(2 \pi \lambda)^{N} \prod_{i=1}^{N} r_{i} d r_{1} d r_{2} \ldots d r_{N} \\
& \underline{r_{i}^{2}=x_{i} \int_{0}^{\infty} \int_{0}^{r 3} \ldots \int_{0}^{r_{2}} \exp \left(-\frac{S N R_{m} \cdot \sigma_{n o i}^{2}}{P_{B S} \cdot A_{1} \cdot \sum_{i=1}^{N} x_{i}^{-\gamma / 2}}\right) \exp \left(-\lambda \pi x_{N}\right)(\pi \lambda)^{N} d x_{1} d x_{2} \ldots d x_{N}}
\end{aligned}
$$

Given a fixed SNR threshold $S N R_{m}$ for multicast services, the corresponding average success probability (or coverage) has been obtained, Moreover, the pdf of SNR in the cell $f(S N R)$ can also be obtained by derivation of the cumulative distribution function of SNR.

\section{Performance evaluations}

The system parameters are shown in Table I where the radius $R$ of $\mathrm{HN}$ is $1000 \mathrm{~m}$. The coverage threshold for multicast is set to be $95 \%$, which means that more than $95 \%$ MS should successfully receive the data for multicast services.

Table I: System Parameters

\begin{tabular}{|l|l|}
\hline Carrier Frequency & $2.5 G$ \\
\hline Frequency Band $B$ & $10 M$ \\
\hline $\begin{array}{l}\text { Path Loss from BS to } \\
\overline{\mathrm{UE}}_{B S}(d B)\end{array}$ & $17.39+37.6 \log _{10}(d[\mathrm{~m}])$ \\
\hline Path Loss parameter $\gamma$ & 3.76 \\
\hline Noise Power Spectrum $N_{0}$ & $-169 \mathrm{dBm} / \mathrm{Hz}$ \\
\hline Cell Radius R & $1000 \mathrm{~m}$ \\
\hline BS power & $46 \mathrm{dBm}$ \\
\hline Coverage for multicast & $95 \%$ \\
\hline
\end{tabular}

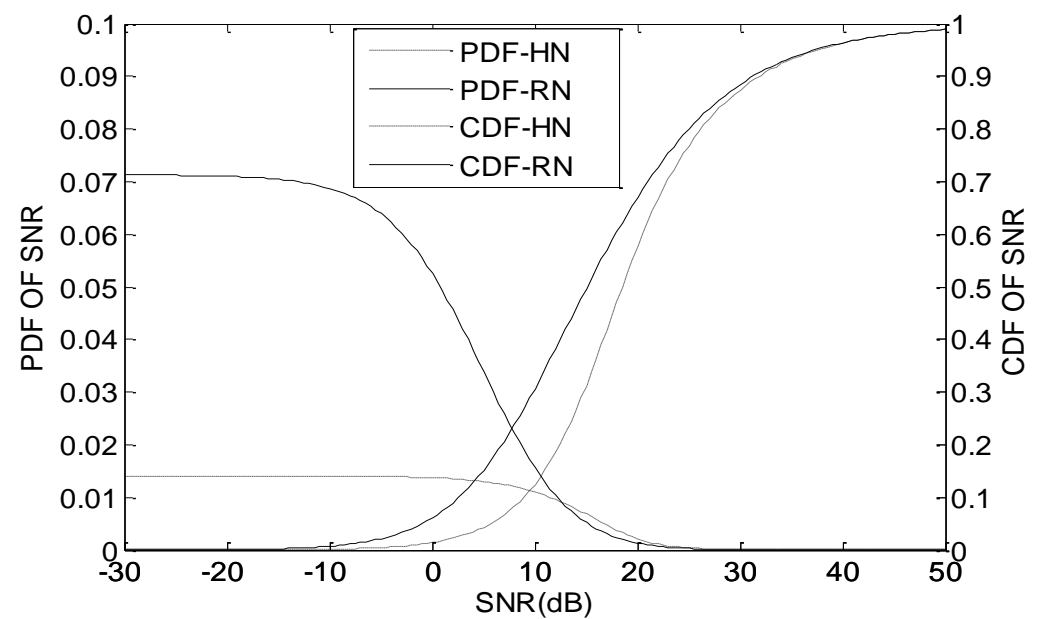

Figure 2. SNR distribution comparision in $\mathrm{RN}$ and $\mathrm{HN}$ without cooperaitive muticast 
Firstly, the pdf and cdf of SNR in single cell is shown in Fig2 where the BS power is set to be a $46 \mathrm{dBm}$. It can be seen that the pdf has a high value in RN when the SNR is low than about $12 \mathrm{~dB}$. Particularly, when SNR is below $0 \mathrm{~dB}$, the pdf value in $\mathrm{RN}$ and $\mathrm{HN}$ are 0.07 and 0.015 respectively, the value in $\mathrm{RN}$ is almost fifth times as that in RN, which means the number of MS with SNR low than $0 \mathrm{~dB}$ will be fifth times as that in HN. Therefore, RN has a poor performance than HN. The same conclusions could also be obtained through cdf curves where a huge gap could be seen. The reason is that $\mathrm{HN}$ could provide more uniform coverage because of the regular deployment of BS.

Next, given the same multicast rate and aiming to provide the same coverage $95 \%$, the BS power needed in HN and RN are shown in Fig. 3 as a function of multicast rate ${ }^{R_{m}}$. It can be seen the BS power needed increased with the multicast data rate. Without cooperative multicast, when the data rate is set to be $1.2 \mathrm{bps} / \mathrm{Hz}, 14 \mathrm{~W}$ will be sufficient to ensure the coverage in $\mathrm{HN}$ while $\mathrm{RN}$ needs $66 \mathrm{~W}$. However, the BS power significantly reduced to $10 \mathrm{~W}$ and $42 \mathrm{~W}$ respectively only with two cooperative BSs, whereas $36.4 \%$ and $28.6 \%$ power are saved compared with the sceneries without cooperation. Thus great improvement can be obtained through cooperation and RN has a larger power reduction through cooperation. Moreover, it can be seen that given the same BS power and BS density, HN has a better performance than RN; therefore, aiming to be energy efficient as $\mathrm{HN}$, large number of the cooperative BSs with low power should be deployed in RN to take advantage of path loss gain and diversity gain. Therefore the total energy could possibly be reduced.

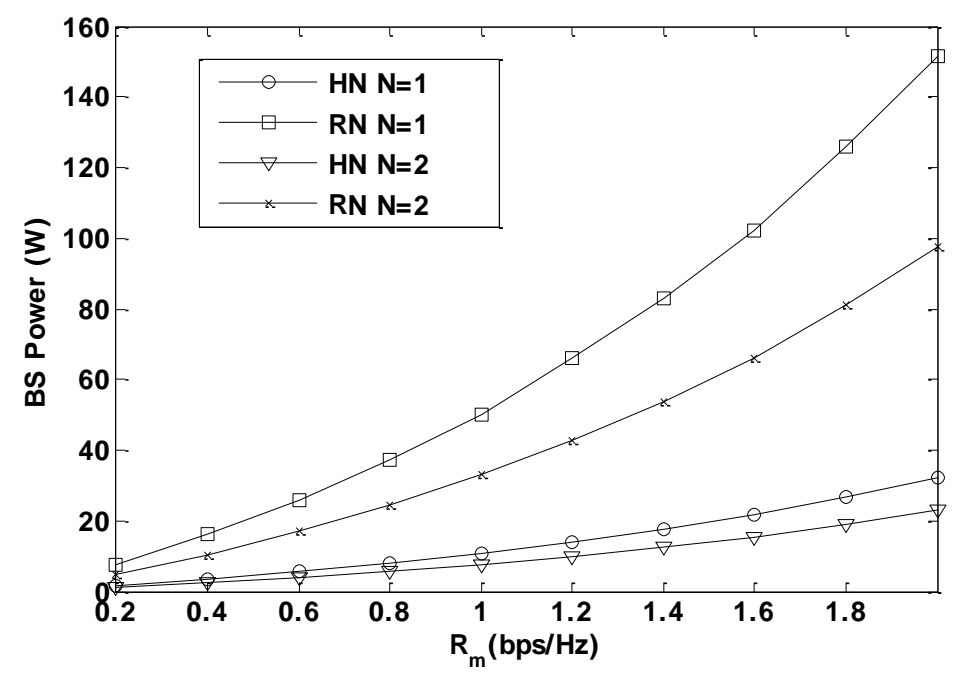

Figure 3. BS power needed to ensue coverage as a function of multicast rate.

\section{Conclusions}

This paper investigated the BS cooperative multicast in $\mathrm{HN}$ and $\mathrm{RN}$, where $\mathrm{CP}$ combining signal processing is employed. The following conclusions are drawn:

The close expression of BS cooperative multicast in $\mathrm{HN}$ and $\mathrm{RN}$ has been obtained such that the performance could be evaluated through numerical expression. The coverage is significantly increased through cooperation in $\mathrm{HN}$ and $\mathrm{RN}$ and the power needed to ensure a given coverage decreased by $30 \%$ when two BSs would like to attend the cooperative multicast.

Aiming to be energy efficient as HN, large number of the cooperative BSs with low power should be deployed in RN to take advantage of path loss gain and diversity gain.

\section{References}

[1] Ericson Mobility Report,June 2013, http://www.ericsson.com/mobility-report.

[2] The Strategy Analytics forecast, "Handset Data Traffic (2001-2017)".

[3] J.G. Andrews, F. Baccelli, and R. K. Ganti. "A tractable approach to coverage and rate in cellular networks", IEEE Trans on Commun, vol. 59(11), pp. 3112-3134, 112011. 
[4] J.G. Andrews, "Seven ways that HetNets are a cellular paradigm shift.", IEEE Commun Mag , vol.51(3), pp. 136-144, 2013.

[5] W. Cheung, T. Quek, and etc, "Throughput Optimization, Spectrum Allocation, and Access Control in Two-Tier Femtocell Networks," IEEE JSAC., vol. 30, no. 3, pp. 561-574, Apr. 2012.

[6] Y. S. Soh, T. Q. S. Quek, M. Kountouris, and H. Shin, "Energy Efficient Heterogeneous Cellular Networks," IEEE J. Select. Areas Commun., vol. 31, no. 5, pp. 840-850, May 2013.

[7] K. Huang and J.G. Andrews, "A stochastic-geometry approach to coverage in cellular networks with multi-cell cooperation”, IEEE ICC, 2011.

[8] V.Garcia, Y.Zhou, J.Shi, "Coordinated Multipoint Transmission in Dense Cellular Networks with User-Centric Adaptive Clustering", submitted to IEEE trans on wireless commun, June 2013.

[9] P. Marsch and G. Fettweis. "Static clustering for cooperative multi-point (comp) in mobile communications.” IEEE ICC , 2011.

[10] Cisco Visual Networking Index: Global Mobile Data Traffic Forecast Update, 2012-2017

[11] 3GPP Tech. Spec. TS 25.346, "Introduction of the Multimedia Broadcast/Multicast Service (MBMS) in the Radio Access Network (RAN)," Mar. 2006.

[12] H. Zhu and J. Wang, "Chunk-based Resource Allocation in OFDMA Systems - Part II: Joint Chunk, Power and Bit Allocation," IEEE Trans. Commun., vol. 60, no. 2, pp. 499-509, Feb. 2012.

[13] S. Lee, Y. Tcha, S. Seo, S. Lee, "Efficient Use of Multicast and Unicast Channels for Multicast Service Transmission," IEEE Trans on Commun, vol. 59, no. 5, p.1264-1267, May 2011.

[14] D. Moltchanov. "Distance distributions in random networks." Ad Hoc Networks, vol. 10, pp. 1146-1166, 2012. 